\title{
A Method to Update the Application Version in Cloud-hosted Virtual Desktops Under Desktop Pool Environment
}

\author{
Jin Gu, Yuan Yuan, Bao Wang \\ CIO Organization of BOE Technology Group Co., Ltd., Beijing, China.
}

\begin{abstract}
With the development of information technology, enterprise users have higher and higher demand for IT terminal system. Meanwhile virtualization technology is having a breakthrough. As an emerging technology as well as a representative of virtualization technology the cloud-hosted virtual desktops technology has been developing rapidly and is widely used in manufacturing enterprises in recent years because of the convenience for management it brought to IT administrators. The realization of the cloud-hosted virtual desktops environment depends on large-scaled use of desktop pools or linked clone technology. However, the above ways are not perfectly compatible with an application release method called Click once which is often adopted by MES production system deployment in the factory, resulting in frequent and repeating download behaviors of applications which created unnecessary waste of IT Infrastructure resources. This paper studies the current situation and proposes an innovative method to update the application in cloud-hosted virtual desktops under desktop pool environment based on the characteristics of business process.
\end{abstract}

Keywords: Virtualization, Cloud-Hosted, Compatible, Large-Scaled.

\section{Introduction}

In recent years, server virtualization and cloud-hosted virtual desktops virtualization technology have been significantly developed, and cloud-hosted virtual desktops technology is increasingly used in large enterprises. As the most popular newly-developing desktop terminal solution over the years, cloud-hosted virtual desktops have made a huge upsurge in enterprise users' office because of its advantage in convenient management and information security. However, we have found that some applications which were suitable for single PC mode has caused a decline of user experience in cloudhosted virtual desktops under desktop pool environment. This paper studies one set of the key applications and provides an effective solution to the compatibility problem by independently developing PowerShell scripts.

\section{Related Technologies}

\subsection{Common Cloud-Hosted Virtual Desktops Delivery Mode -Sharing Mode in Desktop Pool (Clone Mode)}

Cloud-hosted virtual desktops separate the front-end I/O and display devices and the back-end computing and storage devices of the traditional desktop working mode. It focuses on managing backstage resources, providing only operating equipment such as displays, mouse and keyboards to users. Cloud-hosted virtual desktops are an emerging computer technology that uses backstage resources remotely through dedicated network protocols. All cloud-hosted virtual desktops vendors can provide a variety of desktop delivery ways, such as delivering a complete and totally independent desktop for a single user or delivering a desktop pool consists of multiple identical desktops replicated from a single operating system template for a number of users, each one will be randomly assigned a desktop when logging in. This paper uses the PVS mode desktop of Citrix as an example to study.

In PVS desktop delivery mode, the administrator needs to maintain an operating system template which is called the golden image. Through the image, you can copy a number of virtual desktops to form a desktop pool. When a user in the corresponding desktop delivery group logs in, the Citrix DDC server will randomly select one desktop from current available virtual desktops in the pool and deliver it to the user. After the user logged out, the desktop will be automatically restored to the initial gold image state. In this mode, there are two features: 
(1) Any configuration or update related to the operating system or registry will be lost after logout, because the virtual desktop will be automatically restored to the initial golden image state;

(2) After login, the virtual desktop will be randomly assigned by the background system and cannot be selected independently. Cloud-hosted virtual desktops software backstage automatically and randomly distributes virtual desktops from all current available ones in the pool.

\subsection{Applications that are critical to the Enterprise}

In the enterprise, there are some necessary software applications for product production and enterprise operation and management. These applications may have already existed and been in use before the deployment of the cloud-hosted virtual desktops or may be put into use after the deployment. All in all, in whatever desktop mode, these applications should be guaranteed to be usable and easy to use. Here is an example of MES software in manufacturing enterprises.

(1) MES system in manufacturing enterprises

Manufacturing Execution System (MES) is an important part of the enterprise information System. The information interaction between the top plan management system and the underlying industrial control system depends on it. In this case, the MES system contains more than 10 software applications, which are green themselves and will be frequently updated during use. To facilitate updates, the application provider deploys the ClickOnce method based on .net.

ClickOnce technical function introduction

ClickOnce is a deployment method included in Microsoft Visual Studio. By using ClickOnce, selfupdatable applications that check for updates every time before the application runs can be created in Windows

In production environment, MES applications' version updates very frequently. Every time there comes an application update, the administrator only needs to release the latest version of application on the Portal page, so ClickOnce enables users to upgrade it on demand. When the user double-clicks the application icon, it will check the latest version first. If there is an updated version on the server, the user will be prompted to download the upgrade and install it before the application can be used.

This method is effective and easy to use in traditional single PC mode. However, in desktop pool mode, a problem arises because that the version number of local application cannot be saved.

\subsection{In PVS Virtual Desktop Environment, You Need to Download the Application Every Time}

As mentioned above, once a user logs off, the desktop he uses will restore to the golden image template state, and at the next login, a random desktop will be delivered.

The certificate information of the MES applications which are published by ClickOnce is a random string generated in the registry. So, after the user's logging off, the certificate information will be invalidated. In other words, when running the program, even if the user has installed the latest version of the application, the server will still assume that the version is too low and the full program needs to be re-downloaded.

Due to the settings of the cloud-hosted virtual desktops power policy, desktops in the pool will be forcibly restored to initial golden image state when being idle for a certain time every day. Then the above phenomenon will occur frequently, not only reducing the end-user experience, but also increasing the network transmission and server overhead.

\section{A Method for Updating Application Version in Cloud-Hosted Virtual Desktops Under Desktop Pool Environment}

Earlier, we've explained that deploying application updates via Clickonce in cloud-hosted virtual desktops under desktop pool environment can result in frequent downloads from the endpoint, so we propose a new solution which deals the updating problem not by Clickonce, but by the use of script codes, which enables us to extract the local program version number and compare it with the serverside program version number to decide whether an updated version program needs to be downloaded. 
Since this judging method extracts version number information from the NAS storage which has nothing to do with such situations as random login in desktop pools or operation system restore occasions, so that the problem can be avoided.

The tools we need to use here are Microsoft AD domain group policy login scripts, Powershell scripts, and MES application publishing server. The implementation step is firstly developed the Powershell login script, and then make it come into effect by setting it in AD domain group policy, in order to realize that when a user logs in, it can automatically check the application program version and update it if there is new on server. At the same time, this script is invoked by the MES portal program. While still online, users can also click to update manually, avoiding the incompatibility between Clickonce release method and the desktop pool mode of cloud-hosted virtual desktops. By negotiating with the MES application provider and reaching an agreement of the new business mode, which is technically base on Powershell scripts as well as the management of the Group Policy in AD domain, we realized the silent upgrade without the users' awareness, and eliminated the need for repeated and repeated upgrades.

The script code logic is as follows:

Check whether the folder of a specific application (in this case, a MES related application) exists, and whether the user is assigned to the application distribution group. If no target folder exists locally, and the user is in the group, copy the application.

Read the manifest file of the user's local program and extract the version number. For example, 1.0.0.7 is the version number in the <asmv1: assembly Identity version $=" 1 \cdot 0.0 .7 "$ line. There is a same manifest file on the server-side, version number will continuously increase as the application updates.

Read the version number of the latest application program on the server side and compare it with the local version number. If the two numbers differ, then it indicates that an updated version of the application has been released, and the latest version needs to be copied. If the two numbers are same, no copy is needed.

\section{Implementation Effect}

We put this solution into our production environment and the results of the operation are exactly as expected:

(1) Read the local program version number through the script, compare it with the server version, update if it is not the latest. While it is the latest, no actions will be taken, which fully adapts to the cloud-hosted virtual desktops environment.

(2) Each MES application has an independent script corresponding to it. Through the AD security group filtering function, users can be assigned different strategies based on their actual needs, reducing concurrent traffic.

(3) The script runs silently, and even if the user installs multiple programs and the programs needs to be updated at the same time, it also hardly affects the system login speed.

In the subsequent production environment, we plan to promote this solution to other application software version update solutions to reduce the user satisfaction drop and network traffic resource waste caused by frequently downloading updates.

\section{Summary}

This paper describes a case in which emerging technologies such as pooling desktops of cloudhosted virtual desktops are not perfectly compatible with existing key production applications as they reform existing IT management models and proposes a workable solution which avoids repeating application downloads and improves user efficiency through innovative IT technologies. 


\section{References}

[1]. Li Haochen, Guo Hongling, Gao Ting. Design and implementation of MES human-computer interaction program based on ClickOnce technology. Manufacturing Automation, 2013, (15).

[2]. Wang Qiang. Smart Client Deployment Technology-ClickOnce Research. Science and Technology Information. 2007,15. 